\title{
The evolution of the photometric properties of Local Group dwarf spheroidal galaxies
}

\author{
F. Calura ${ }^{1}$, G. A. Lanfranchi ${ }^{2,3}$, and F. Matteucci ${ }^{1,3}$ \\ 1 INAF - Osservatorio Astronomico di Trieste, via G. B. Tiepolo 11, 34131 Trieste, Italy \\ e-mail: fcalura@oats.inaf.it \\ 2 Núcleo de Astrofísica Teórica, CETEC, Universidade Cruzeiro do Sul, R. Galvão Bueno 868, Liberdade, 01506-000 São Paulo, SP, \\ Brazil \\ 3 Dipartimento di Astronomia - Universitá di Trieste, via G. B. Tiepolo 11, 34131 Trieste, Italy
}

Received 10 August 2007 / Accepted 11 January 2008

\begin{abstract}
Aims. We investigate the present-day photometric properties of the dwarf spheroidal galaxies in the Local Group. From the analysis of their integrated colours, we consider a possible link between dwarf spheroidals and giant ellipticals. From the analysis of the $M_{V}$ vs. $(B-V)$ plot, we search for a possible evolutionary link between dwarf spheroidal galaxies (dSphs) and dwarf irregular galaxies (dIrrs). Methods. By means of chemical evolution models combined with a spectro-photometric model, we study the evolution of six Local Group dwarf spheroidal galaxies (Carina, Draco, Sagittarius, Sculptor, Sextans and Ursa Minor). The chemical evolution models, which adopt up-to-date nucleosynthesis from low and intermediate mass stars as well as nucleosynthesis and energetic feedback from supernovae type Ia and II, reproduce several observational constraints of these galaxies, such as abundance ratios versus metallicity and the metallicity distributions. The proposed scenario for the evolution of these galaxies is characterised by low star formation rates and high galactic wind efficiencies.

Results. Such a scenario allows us to predict integrated colours and magnitudes which agree with observations. Our results strongly suggest that the first few Gyrs of evolution, when the star formation is most active, are crucial to define the luminosities, colours, and other photometric properties as observed today. After the star formation epoch, the galactic wind sweeps away a large fraction of the gas of each galaxy, which then evolves passively.

Our results indicate that it is likely that at a certain stage of their evolution, dSphs and dIrrs presented similar photometric properties. However, after that phase, they evolved along different paths, leading them to their disparate present-day properties.
\end{abstract}

Key words. galaxies: dwarf - galaxies: evolution - galaxies: Local Group

\section{Introduction}

The dwarf spheroidal galaxies (dSphs) are among the most common types of galaxies in the universe. They are found normally in groups (Côté et al. 1997) and clusters (Phillips et al. 1998; Ferguson \& Sandage 1991). The ones found in the Local Group have become an increasingly important matter of study in the last few years due to their proximity, which enables one to study in detail objects and processes which were formally restricted to our own Galaxy.

Several issues regarding the formation and evolution of the Local Group dSphs can help in the attempt to clarify the whole subject of galaxy formation. For example, how did the dSphs form? When? What mechanism ruled their evolution? Are these galaxies remnants of the building blocks from which larger galaxies assembled? Is their evolution mainly affected by the environment or do internal processes play a major role? Are these galaxies linked to other types of dwarf galaxies in the context of any evolutionary scenario? In order to answer these questions, one should try to understand not only the present day properties of the dSphs, but also try to understand their past evolutionary history. One possible procedure is to make use of models which, being based on present day observational constraints, allow one to trace the past evolution of the dSphs.
Originally, the local dSphs were believed to be very old simple systems, similar to globular clusters (Shapley 1938). However, more recent deep photometric observations of main sequence turn-off stars revealed also intermediate-age populations, as well as a significant metallicity range, different from most globular clusters. In fact, analysis of colour-magnitude diagrams (CMDs) suggested that these galaxies are characterised by complex and different star formation (SF) histories (van den Bergh 1994; Hernandez et al. 2000; Dolphin et al. 2005). In almost all cases, the mechanisms which trigger and control the SF are yet unknown and several scenarios have been proposed. These scenarios should also explain the complete lack of gas in these galaxies, the low metallicities, the low values of $[\alpha / \mathrm{Fe}]$ relative to Galactic stars with the same $[\mathrm{Fe} / \mathrm{H}]$ (Bonifacio et al. 2000; Shetrone et al. 2001, 2003; Tolstoy et al. 2003; Bonifacio et al. 2004; Venn et al. 2004; Sadakane et al. 2004; Geisler et al. 2005; Monaco et al. 2005) and the metallicity distributions, including the large metallicity range (Koch et al. 2005; Bellazzini et al. 2002).

Several attempts to model the properties of dSphs have been performed, generally following different approaches. By means of high-resolution cosmological numerical simulations, Ricotti \& Gnedin (2005) and Kawata et al. (2006) studied dSphs in 
a cosmological framework. Their studies are useful to understand the link between the dSphs and the first galaxies, but cannot investigate in detail the chemical properties of dSphs and the relative roles of SNe Ia and II in the chemical enrichment and gas ejection processes. Marcolini et al. (2006), by means of a chemo-dynamical evolution model, studied the evolution of the interstellar medium (ISM) of the Draco dSph. In their picture no galactic wind develops and, in order to deplete the galaxy of its gas and to stop star formation, one must invoke an external mechanism, such as ram pressure stripping or tidal interactions. Fenner et al. (2006) and Ikuta \& Arimoto (2002) studied the properties of dSphs by means of chemical evolution models including galactic winds. Their results indicate that a small fraction of the ISM is carried away by the $\mathrm{SN}$-driven winds and confirm the need of external gas removing processes to reproduce the present-day gas fractions of dSphs (see also Mori \& Burkert 2000; Mayer et al. 2006). Lanfranchi \& Matteucci (2003, 2004, hereinafter LM03, LM04), alternatively, by means of a detailed chemical evolution model with galactic winds, were able to reproduce many observational constraints of six local dSph galaxies (Carina, Draco, Sagittarius, Sculptor, Sextans, Ursa Minor). The scenario proposed by these authors considered low efficiency star formation rates (SFR) derived from colour-magnitude diagrams together with intense galactic winds.

LM03, 04 were able to reproduce not only the chemical properties, but also the lack of central neutral gas and the metallicity distributions of the studied galaxies. In particular, the $[\alpha / \mathrm{Fe}]$ vs. $[\mathrm{Fe} / \mathrm{H}]$ and neutron capture element ratios in these galaxies are well reproduced as well as the stellar metallicity distribution (SMD).

The photometric properties of the local dSphs, however, are rarely addressed in any of these models, even though they could help, not only in constraining the formation and evolution of these galaxies, but also in clarifying the subject of a possible evolutionary connection between the gas poor dSph galaxies and gas rich dwarf irregular galaxies (dIrrs). In such a scenario, a starburst in a dIrr gives rise to a super wind which removes all the gas of the galaxy (which could be removed also by ram pressure stripping or tidal stripping) and halts the SF, giving rise to a dSph galaxy (Lin \& Faber 1983; Dekel \& Silk 1986; van den Bergh 1994; Papaderos et al. 1996; Davies \& Phillipps 1988). There are, however, several drawbacks in that scenario, from both the chemical and photometric point of view. First, the large scale distribution of dSphs is substantially different from that of dIrrs. Most of the dSphs cluster around the two giant spirals of the Local Group (Grebel 1998), with very few exceptions. On the other hand, most of the dIrrs lie at large (>500 kpc) distances from the large galaxies (Mateo 1998). This is probably linked to the morphology-density relation for dwarf galaxies and is interpreted as an evidence of environmental effects on galaxy evolution (Grebel 2001). In particular, it may be possible that their proximity to large galaxies had some effects on the evolution of dSphs.

Futhermore, the dIrrs and dSphs show rather different observational properties. The integrated colour-magnitude diagram of the Local Group dwarf galaxies exhibits a clear distinction between the dSphs and the dIrrs, which occupy different regions (Mateo 1998). A few galaxies, namely transition type dwarfs, exhibit intermediate photometric properies between those of dSphs and dIrrs. Also the luminosity/metallicity relation can be used to distinguish between dIrrs and dSphs at the present epoch (Mateo 1998; Grebel et al. 2003). At the same luminosity, the dIrrs tend to exhibit lower metallicities than the dSph galaxies. These two facts could be related to different evolutionary histories for the two types of dwarfs and only a study of their past history could help in clarifying this issue.

In this work we show that a spectro-photometric code coupled with a chemical evolution model allows us to investigate the past evolution of the dSphs and impose constraints on the formation and evolution of these galaxies and also to the possible connection with other types of dwarf and giant galaxies. A chemical evolution code which reproduces successfully several observational constraints can provide the parameters required to predict the evolution of the photometric properties of a sample of six local dSph galaxies.

The paper is organized as follows: in Sect. 2, the chemical evolution models which reproduce the chemical data of these galaxies and their results are described. In Sect. 3 we describe the spectro-photometric code. The results of our models compared to observational data are shown in Sect. 4 and finally in Sect. 5 we draw some conclusions.

\section{Chemical evolution models}

In order to provide the star formation rates, metallicities, gas mass, HI mass and predictions of other parameters useful to the spectro-photometric code, we adopted the same chemical evolution models as described in our previous works. In particular, the models for Draco and Ursa Minor are taken from Lanfranchi \& Matteucci (2007 - LM07), Carina and Sagittarius from Lanfranchi et al. (2006b - LMC06b), Sculptor from Lanfranchi et al. (2006a - LMC06a) and Sextans from LM04. The main parameters adopted for the model of each galaxy can be seen in Table 1. In the first column of Table 1, we list the six dSphs studied in this paper. $M_{\mathrm{tot}}^{\text {initial }}(\mathrm{Col} .2)$ is the baryonic initial mass of the galaxy, in $M_{\odot} . w_{i}(\mathrm{Col} .3)$ is the wind efficiency. $v(\mathrm{Col} .4)$ is the star-formation efficiency, a quantity expressing the intensity of each star formation event and is expressed in $\mathrm{Gyr}^{-1}$. Throughout this paper, $v$ is used to compute the fiducial SFRs. $v_{1}$ and $v_{2}$ (Col. 5) are the lower and uper limits on the SF efficiency, as shown in LM04. $v_{1}$ and $v_{2}$ are used in Sects. 4.4 and 4.5 to provide the errors associated with the theoretical predictions of the present-day colours of dSphs. Finally, the IMF (Col. 6) is the stellar initial mass function.

For more details on the models, we refer the reader to LM03, LM04. All these models already reproduced very well the $[\alpha / \mathrm{Fe}]$ ratios, the $[\mathrm{Ba} / \mathrm{Fe}]$ and $[\mathrm{Eu} / \mathrm{Fe}]$ ratios, the stellar metallicity distributions and the present total mass and gas mass observed in these galaxies. In these models, we adopted a scenario in which the dSph galaxies form through a continuous and fast infall of pristine gas. We assume that the star formation histories (SFHs) of dSph galaxies consist of one or more episodes of different duration. The main parameters in determining the star formation history consist in the burst duration $t_{\mathrm{B}}$, the number of the bursts $n$ and the star formation efficiency $v$. The burst duration $t_{\mathrm{B}}$ and the number of the bursts $n$ are provided by the observed colour-magnitude diagrams (CMDs), representing the most reliable constraints on the star formation history of each dSph (Dolphin 2002; Dolphin et al. 2005). These diagrams suggest a unique long $(t>3 \mathrm{Gyr})$ episode of SF in Draco, Sextans, Sculptor, Ursa Minor and Sagittarius (Dolphin et al. 2005; Carrera et al. 2002; Aparicio et al. 2001; Hernandez et al. 2000) and a few episodes (4) in Carina (Rizzi et al. 2003). It is worth mentioning that the models adopting the SFHs taken from observed CMDs (as described in Table 2) reproduce very well the abundance ratios observed in these $\mathrm{dSph}$ galaxies. More important, the adopted SFHs for Draco, Ursa Minor and 
Table 1. Main parameters for the models of the six dSph galaxies studied in this paper.

\begin{tabular}{lccccc}
\hline \hline Galaxy & $M_{\text {tot }}^{\text {initial }}\left(M_{\odot}\right)$ & $w_{i}$ & $v\left(\mathrm{Gyr}^{-1}\right)$ & $v_{1}, v_{2}\left(\mathrm{Gyr}^{-1}\right)$ & IMF \\
\hline Sextans & $5 \times 10^{8}$ & 9 & 0.08 & $0.01-0.3$ & Salpeter \\
Sculptor & $5 \times 10^{8}$ & 13 & 0.2 & $0.05-0.5$ & Salpeter \\
Sagittarius & $5 \times 10^{8}$ & 9 & 3.0 & $1.0-5.0$ & Salpeter \\
Draco & $5 \times 10^{8}$ & 4 & 0.03 & $0.005-0.1$ & Salpeter \\
Ursa Minor & $5 \times 10^{8}$ & 10 & 0.2 & $0.05-0.5$ & Salpeter \\
Carina & $5 \times 10^{8}$ & 5 & 0.15 & $0.02-0.4$ & Salpeter \\
\hline
\end{tabular}

Carina produced SMDs in very good agreement with observations (LM07, LMC06b). In the case of Carina, LMC06b tested several models adopting in each one a different SFH (in particular the ones from Smecker-Hane et al. 1996; Hurley-Keller et al. 1998; Hernandez et al. 2000; Dolphin 2002; and Rizzi et al. 2003) and compared the predictions of such models with the $[\alpha / \mathrm{Fe}],[\mathrm{Ba} / \mathrm{Fe}],[\mathrm{Eu} / \mathrm{Fe}]$ and the SMD of Koch et al. (2006). The model with the Rizzi et al. (2003) SFH (the same adopted in this work) was the one which gave the best result.

\subsection{Basic ingredients}

In Table 2, we present the SFHs for the six dSph galaxies modelled in this work, along with the references to the SFHs inferred from studies of the observed CMDs, considered in this paper to compute the SFHs for the fiducial models. In the first column, we indicate the six dSphs. In the second, third and fourth columns, we show the number $n$, the times of occurrence $t_{\mathrm{B}}$ and the durations $d$ of the star formation episodes, as inferred from the observed CMDs, respectively. Finally, in the last column we present the references for the observed CMDs used to determine the SFHs.

The galactic wind is actually one vital ingredient in these models since it plays a crucial role in the evolution of the galaxies. When the thermal energy of the gas equals or exceeds its binding energy, a wind develops (see for example Matteucci \& Tornambé 1987). The thermal energy of the gas is controlled mainly by the thermalization efficiencies of the supernovae explosions $\left(\eta_{\mathrm{SNII}}\right.$ for SNe II and $\eta_{\mathrm{SNIa}}$ for SNeIa) and stellar winds $\left(\eta_{\mathrm{SW}}\right)$, which control the fraction of the energy converted to thermal energy of the gas (see Bradamante et al. 1998). The binding energy of the gas, on the other hand, is strongly influenced by assumptions concerning the presence and distribution of dark matter (Matteucci 1992). A diffuse $\left(R_{\mathrm{e}} / R_{\mathrm{d}}=0.1\right.$, where $R_{\mathrm{e}}$ is the effective radius of the galaxy and $R_{\mathrm{d}}$ is the radius of the dark matter core $)$ but relatively massive $\left(M_{\mathrm{dark}} / M_{\mathrm{Lum}}=10\right)$ dark halo has been assumed for each galaxy. This particular configuration allows the development of a galactic wind in these small systems without destroying them. The galactic winds are intense (the rate of the wind is $4-13$ times the SFR) in the models for the dSph galaxies in order to reproduce the decrease observed in the $[\alpha / \mathrm{Fe}]$ vs. $[\mathrm{Fe} / \mathrm{H}]$ plots, as well as several features of the stellar metallicity distributions (LM07), and also to remove a large fraction of the gas content of the galaxy. A possible justification for intense galactic winds in $\mathrm{dSph}$ galaxies comes from the fact that their potential well is not as deep as in the case of the Dwarf Irregular galaxies and is more extended (Mateo 1998; Guzman et al. 1998; Graham \& Guzman 2003). In that sense, the gas would be able to escape more easily from these systems. These models allow us to follow in detail the evolution of the abundances of several chemical elements and isotopes, starting from the matter reprocessed by the stars and restored into the ISM
Table 2. The adopted star formation histories for the $\mathrm{dSph}$ galaxies studied in this paper.

\begin{tabular}{|c|c|c|c|c|}
\hline & $n$ & $\begin{array}{l}\text { SFHs } \\
t_{\mathrm{B}}(\mathrm{Gyr})\end{array}$ & $d(\mathrm{Gyr})$ & References \\
\hline Sextans & 1 & 0 & 8 & LM04 \\
\hline Sculptor & 1 & 0 & 7 & Dolphin (2002) \\
\hline Sagittarius & 1 & 0 & 13 & Dolphin (2002) \\
\hline Draco & 1 & 0 & 4 & $\begin{array}{l}\text { Aparicio et al. (2001), } \\
\text { Dolphin et al. (2005) }\end{array}$ \\
\hline Ursa Minor & 1 & 0 & 3 & $\begin{array}{l}\text { Carrera et al. (2002), } \\
\text { Dolphin et al. (2005) }\end{array}$ \\
\hline Carina & 4 & $0 / 2 / 7 / 9$ & $2 / 2 / 2 / 2$ & Rizzi et al. (2003) \\
\hline
\end{tabular}

by stellar winds and type II and Ia supernova explosions. The type Ia $\mathrm{SN}$ progenitors are assumed to be $\mathrm{C}-\mathrm{O}$ white dwarfs in binary systems according to the formalism originally developed by Greggio \& Renzini (1983) and Matteucci \& Greggio (1986). Type II SN explosions are assumed to originate from core collapse of single massive $\left(M>8 M_{\odot}\right)$ stars, leaving as remnants a neutron star or a black hole.

The main assumptions of the models are:

- one zone with instantaneous and complete mixing of gas inside this zone;

- no instantaneous recycling approximation, i.e. the stellar lifetimes are taken into account;

- the evolution of several chemical species (H, D, He, C, N, O, $\mathrm{Mg}, \mathrm{Si}, \mathrm{S}, \mathrm{Ca}, \mathrm{Fe}, \mathrm{Ba}$ and $\mathrm{Eu})$ is followed in detail;

- the nucleosynthesis prescriptions include the yields of Nomoto et al. (1997) for type Ia supernovae, Woosley \& Weaver (1995) (with the corrections suggested by François et al. 2004) for massive stars $\left(M>10 M_{\odot}\right)$, van den Hoek \& Groenewegen (1997) for intermediate mass stars (IMS) and for $\mathrm{Ba}$ and $\mathrm{Eu}$ the ones described in LMC06a and Cescutti et al. (2006).

The basic equations of chemical evolution are the same as described in LM03 and LM04 (see also Tinsley 1980; Matteucci 1996), as are the prescriptions for the SFR $\psi(t)$, which follows a Schmidt law (Schmidt 1963):

$\psi(t)=v G^{k}(t)$

where $G(t)$ is the gas fraction with respect to the total initial mass. $v$ is the SF efficiency, namely the SFR per unit mass of gas. For each dSph galaxy, the SF efficiency has been determined in order to best reproduce the observed abundance ratios (LM04) and the SMDs (LMC06b, LM07).

As in previous works (LM03, LM04), we assume $k=1$ and an initial mass function (IMF) from Salpeter (1955). The choice of the Salpeter IMF allows one to reproduce the abundances observed in dwarf galaxies (LM04; Recchi et al. 2002; LM07), as well as their present-day observational features (Calura \& Matteucci 2006). Moreover, as shown by Calura \& Matteucci (2004), the assumption of a Salpeter IMF allows one to account also for the local metal budget.

Our models include also infall and galactic winds, occurring at a rate $\left(\dot{G}_{i}\right)$ and $\left(\dot{G}_{i}\right)_{\text {out }}$, respectively. The rate of gas infall is defined as:

$\left(\dot{G}_{i}\right)_{\mathrm{inf}}=A \mathrm{e}^{-t / \tau}$

with $A$ being a suitable constant and $\tau$ the infall time-scale which is assumed to be $0.5 \mathrm{Gyr}$. 
The rate of gas loss via galactic winds for each element $i$ is assumed to be proportional to the star formation rate at the time $t$ :

$\left(\dot{G}_{i}\right)_{\mathrm{out}}=w_{i} \psi(t)$

where $w_{i}$ is the wind efficiency parameter.

In the left panel of Fig. 1, we show the evolution of the star formation rates for the six dSphs considered in this work. For each galaxy, we show the fiducial star formation rates and the star formation rates computed by assuming lower and upper limits for the star formation efficiencies as in LM04. We will refer to these latter models as minimal and maximal model, respectively. The minimal and maximal models give an idea of the scatter in the SF efficiency parameter and of how wide its range is, within which one can still obtain a reasonably good fit to the abundance pattern observed in dSphs (LM04).

In some cases, it appears that the minimal and maximal lines may reverse. This effect is related to the fact that, in each model, the time when the galactic wind develops depends on the assumed SF efficiency. In Fig. 1, the onset of the wind corresponds to the change in slope of each curve. In general, the lower the $\mathrm{SF}$ efficiency, the later the galactic wind develops. For this reason, the lower the SF efficiency, the longer is the period during which the star formation remains high. This effect is particularly strong for the Draco and Sextans models, since these are the models presenting the widest range of values for $v$.

The lower and upper limits for the SF efficiency are indicated in Table 1 as $v_{1}$ and $v_{2}$, respectively. These quantities will be used later in Sects. 4.4-4.5 to derive error bars for the present day colours of dSphs. Unless otherwise stated, all the calculations will be performed by using the fiducial star formation rates, computed using the SF efficiencies $v$ reported in Table 1.

In Fig. 1, right panel, we plot the evolution of the gas fractions (defined as the ratio between the gas mass and the total baryonic mass, i.e. gas plus stars) for the six fiducial dSph models. As the gas is transformed into stars and removed by the winds, the gas fractions of the dSphs decrease by several orders of magnitude in few Gyr. As shown in Eq. (1), at any time, the SFR depends on the amount of available gas present in the galaxy. For this reason, the star formation continues even after the onset of the wind, but at a considerably lower rate. In the few last Gyr of evolution, the oscillations in the gas content are due to injection of gas into the ISM from dying stars and to gas removal via winds and star formation.

Fenner et al. (2006) and Ikuta \& Arimoto (2002) suggested that galactic winds do not play a major role in removing the gas from dSphs. In order to explain the lack of gas in dSph galaxies, they invoked a complementary mechanism, such as tidal stripping or ram pressure. However, by means of closed-box models, Ikuta \& Arimoto (2002) are not able to reproduce the lowest values of $[\alpha / \mathrm{Fe}]$ observed in dSphs without invoking a very steep IMF, whereas our models can reproduce those values very well with a normal Salpeter-like IMF (see also Geisler et al. 2007). Besides that, the works by Fenner et al. (2006) and Ikuta \& Arimoto (2002) do not compare their predictions with the stellar metallicity distributions (SMDs) observed in dSph galaxies. On the other hand, Lanfranchi \& Matteucci (2007) consider the constraints provided by SMDs of local dSph galaxies, arguing that these diagrams can be reproduced only if strong galactic winds are taken into account.

Recent evidence (Tolstoy et al. 2004) is finding that the Sculptor dSph has at least two distinct populations of stars, with different metallicity, spatial distributions, and kinematics.
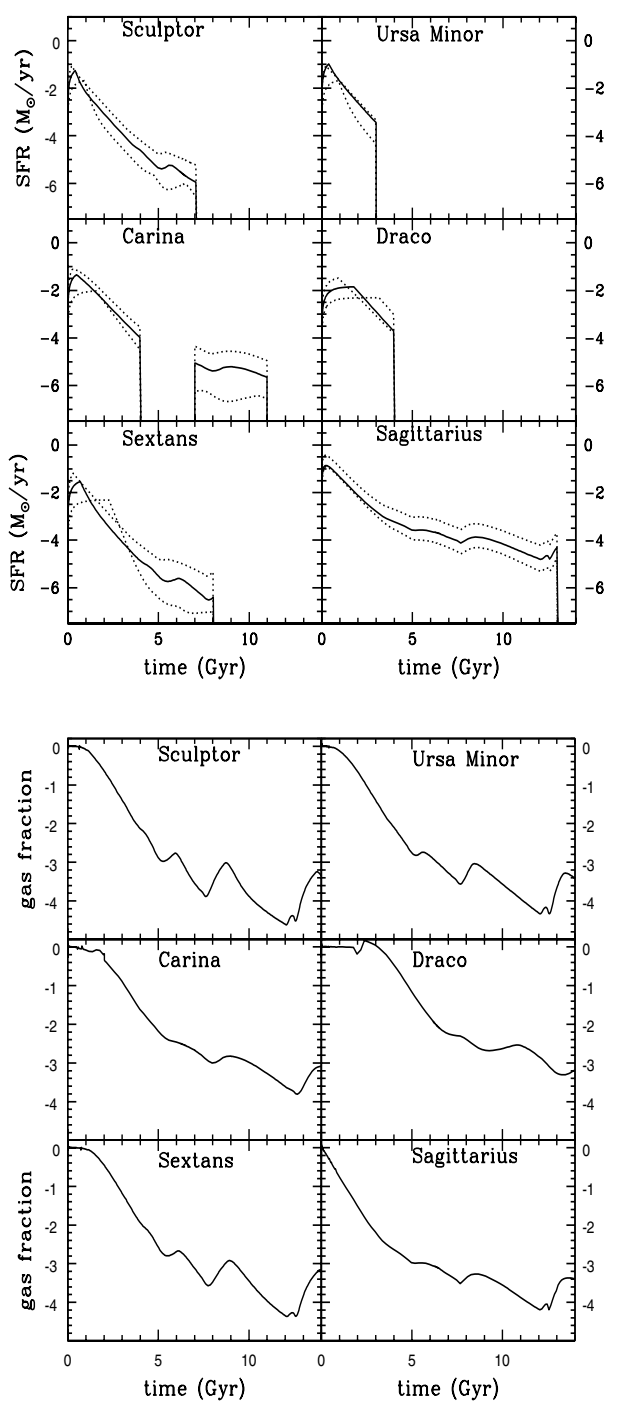

Fig. 1. Upper panel: evolution of the SFR as a function of time for the six dSphs. The solid lines represent the SFRs of the fiducial models. The dotted lines are star formation histories computed by assuming lower and upper limits for the star formation efficiencies, as indicated by the quantities $v_{1}$ and $v_{2}$ of Table 1 , respectively. Lower panel: evolution of the gas fractions for the fiducial models of the six dSphs (solid lines).

These two different stellar populations can be explained as the consequence of a monolithic collapse of gas, with more metal rich stars forming in the central regions of the galaxy, and metal poor stars in the outskirts. Since our model is one-zone, we cannot distinguish between these two populations. However, we are able to reproduce quite well the overall chemical features for the dSphs. Furthermore, a photometric study of the spatial distribution of the stellar populations is beyond the aims of this paper, since here we are interested in the photometric properties of each dSph as a whole and not as a function of radius.

\section{The spectro-photometric code}

To calculate galaxy colours and magnitudes, we use the photometric code by Bruzual \& Charlot (2003, hereinafter BC). This code allows one to compute the time evolution of the spectra of stellar populations in the metallicity range $0.0001 \leq Z \leq 0.05$, across the whole wavelength range from $3200 \AA$ to $9500 \AA$. We implemented the $\mathrm{BC}$ code by taking into account the evolution 
of metallicity in galaxies (Calura et al. 2004). To model the spectral evolution of the $6 \mathrm{dSph}$ galaxies studied in this paper, we use simple stellar population (SSP) models calculated for a Salpeter (1955) IMF, with lower and upper cutoffs $m_{\mathrm{L}}=0.1 M_{\odot}$ and $m_{\mathrm{L}}=100 M_{\odot}$, respectively. Dust extinction is also properly taken into account. We adopt the "screen" dust geometric distribution which, according to UV and optical observations of local starburst galaxies, is to be considered favored over the "slab" model (Calzetti et al. 1994). The absorbed flux $I_{\mathrm{a}}(\lambda)$ of a stellar population behind a screen of dust is given by (Calzetti 2001):

$I_{\mathrm{a}}(\lambda)=I_{\mathrm{I}}(\lambda) \exp (-\tau(\lambda))$

where $I_{\mathrm{I}}(\lambda)$ represents the intrinsic, unobscured flux at the wavelength $\lambda$.

We assume that the optical depth $\tau(\lambda)$ is proportional to the gas surface density and to the metallicity $Z$, according to:

$\tau(\lambda)=C \frac{k(\lambda) M_{\mathrm{gas}} Z}{\pi r_{\mathrm{d}}^{2}}$

where $k(\lambda)$ is the extinction curve. In this paper, we adopt the extinction curve suggested by Calzetti (1997). $M_{\text {gas }}$ is the total gas mass and $r_{\mathrm{d}}$ is the radius of the galaxy. We assume that, in each $\mathrm{dSph}$ galaxy, the neutral gas follows a homogeneous, spherical distribution within a radius $r_{\mathrm{d}}=0.5 \mathrm{kpc}$. This value is of the same order as the estimated tidal radii of some of these systems (Irwin \& Hatzidimitriou 1995; Gallagher et al. 2003). The constant $C$ in Eq. (5) is chosen in order to reproduce the Milky Way average optical depth in the $V$ band, $\tau_{V}=0.8$ (Calzetti 2001), given a gas surface density of $\sim 10 M_{\odot} \mathrm{pc}^{-2}$ and the solar metallicity (Calura et al. 2004).

\section{Results}

\subsection{The evolution of the $V$ band luminosities}

Each dSph galaxy is assumed to be composed of different stellar populations, each one characterised by a particular metallicity. The metallicity values for the stellar populations are the ones of the $\mathrm{BC}$ spectro-photometric model, i.e. $Z=$ $0.0001,0.0004,0.004,0.008,0.02\left(Z_{\odot}\right), 0.05$. The luminosity of a dSph galaxy is given by the sum of the luminosities of the different SSPs.

In Figs. 2-7 we show the predicted evolution of the luminosities for the different stellar populations, formed at different $Z$, composing the Sculptor, Carina, Sextans, Ursa Minor, Draco and Sagittarius dSphs, respectively. In most of the cases, the total luminosities of the $\mathrm{dSph}$ galaxies are dominated by the SSPs formed at the lowest metallicities, $Z=0.0001$ and $Z=0.0004$. This is true for the Sculptor, Carina, Sextans, Ursa Minor and Draco dSphs. For each galaxy, the stellar population dominating the metallicity distribution (LM04) dominates also the total $K$ and $B$ light. For all of these galaxies, the stellar populations with metallicities $Z>0.0004$ give negligible contributions to the total luminosities. A particular case is represented by the Sagittarius dSphs. In this galaxy, the $B$ and $K$ luminosities are dominated by stellar populations with higher metallicities, i.e. $Z=0.004$ and $Z=0.008$. This is reflecting the fact that the Sagittarius galaxy had a star formation history substantially different from the other $5 \mathrm{dSphs}$ studied in this paper (see Fig. 1). From the chemical evolution point of view, the difference between Sagittarius and the other dSph galaxies studied here resides in the fact that the former is characterised by a star formation efficiency higher ( $>10$ times) than the others. The slow

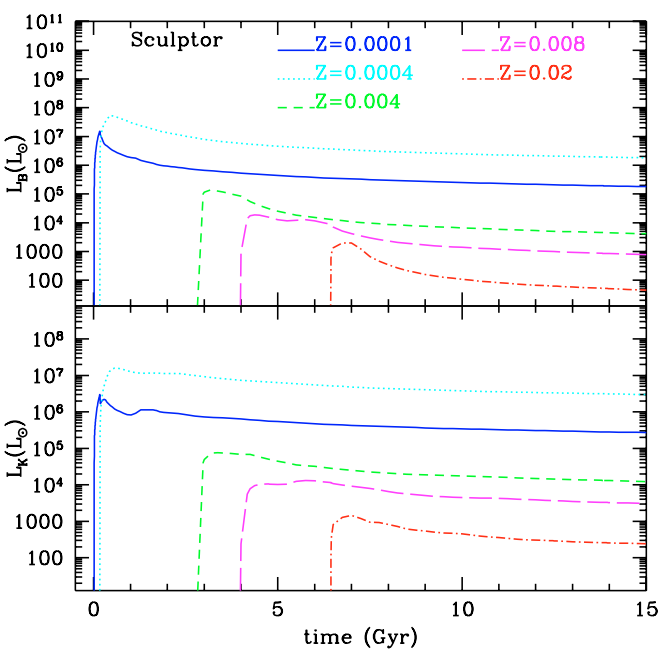

Fig. 2. Predicted $B$ - (upper panel) and $K$ - (lower panel) band luminosities for the different stellar populations of the Sculptor dSph formed at various metallicities as a function of time.

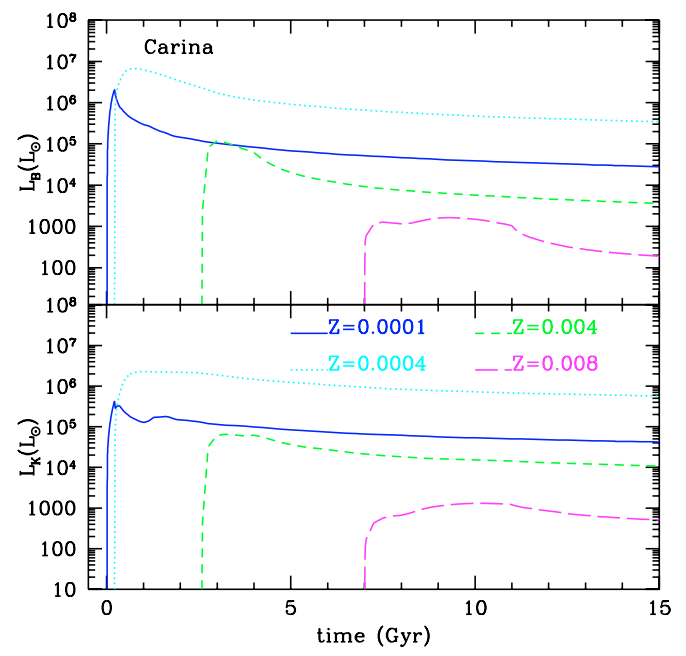

Fig. 3. Predicted $B$ - (upper panel) and $K$ - (lower panel) band luminosities for the different stellar populations of the Carina dSph formed at various metallicities as a function of time.

evolution and the occurrence of an intense galactic wind, which prevents metal rich stars to be formed in a significant number, explains the fact that stellar populations with low metallicities dominate the total luminosity of these other dSph galaxies besides Sagittarius.

The total luminosities of the dSph galaxies in the bluest bands of the optical spectra (i.e. $U, B, V$ ) are affected by dust extinction effects. These effects are negligible at the present time, but they must have played an important role in the past. In Fig. 8, we show the predicted evolution of the $V$ band Luminosities for the six dSph galaxies, both intrinsic and attenuated by dust extinction. For all the galaxies, the effects of dust extinction are noticeable during the star formation phase, when the galaxies have rich reservoirs of gas. After the onset of the galactic winds and the consequent decrease of the star formation, for each galaxy almost all of the interstellar gas is swept away and the effects of extinction become negligible. At the present time (13 Gyr), the $\mathrm{dSphs}$ have very little gas and the intrinsic and attenuated $V$ band luminosities cannot be distinguished. 


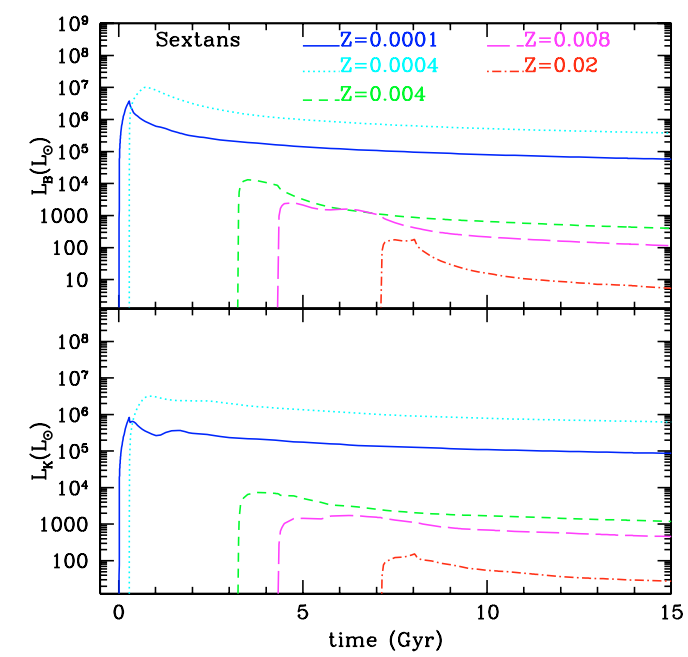

Fig. 4. Predicted $B$ - (upper panel) and $K$ - (lower panel) band luminosities for the different stellar populations of the Sextans dSph formed at various metallicities as a function of time.

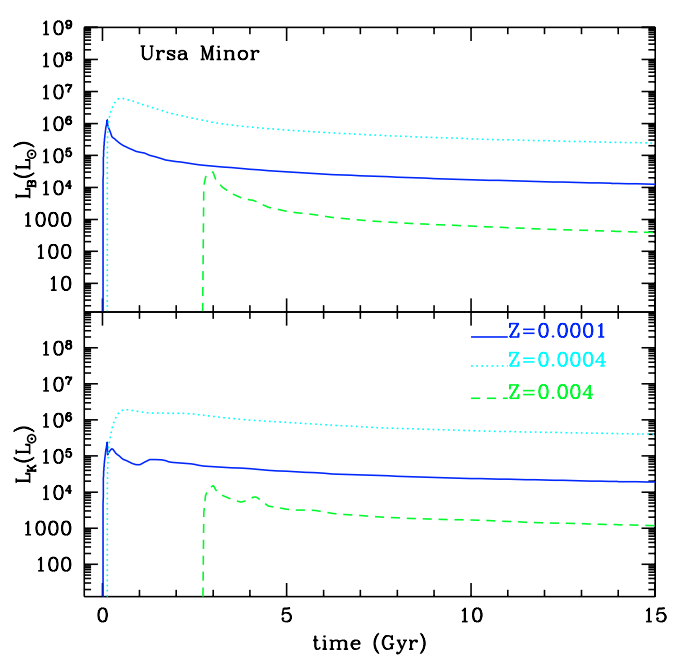

Fig. 5. Predicted $B$ - (upper panel) and $K$ - (lower panel) band luminosities for the different stellar populations of the Ursa Minor dSph formed at various metallicities as a function of time.

\subsection{The evolution of the colours of dSphs}

In Fig. 9, we show the predicted $U-V$ (solid lines) and $V-K$ (dashed lines) colours for the six dSphs. It is possible to see that during the star formation period, in general lasting some Gyr, the attenuated colours are redder than the intrinsic ones. After the end of the star formation, when the galaxies have lost almost all of their gas, dust extinction is negligible and the curves for the attenuated and the intrinsic colours overlap. This relatively short but important period corresponds to the first few Gyr of evolution, exactly the same epoch when the star formation is more intense. As mentioned in LMC06b, the evolution of several dSphs is almost totally determined by the first few Gyr after the beginning of the star formation. In this time interval, the pattern of the abundance ratios and the stellar metallicity distributions are almost totally in place (LMC06b), and also the colours are determined, supporting the idea that the main activity in the dSph galaxies occurred very far in the past and, after that, they evolved passively or with very low SF activity.

Finally, it is worth noting that the curve of the Draco dSph is substantially different from the others. This can be understood

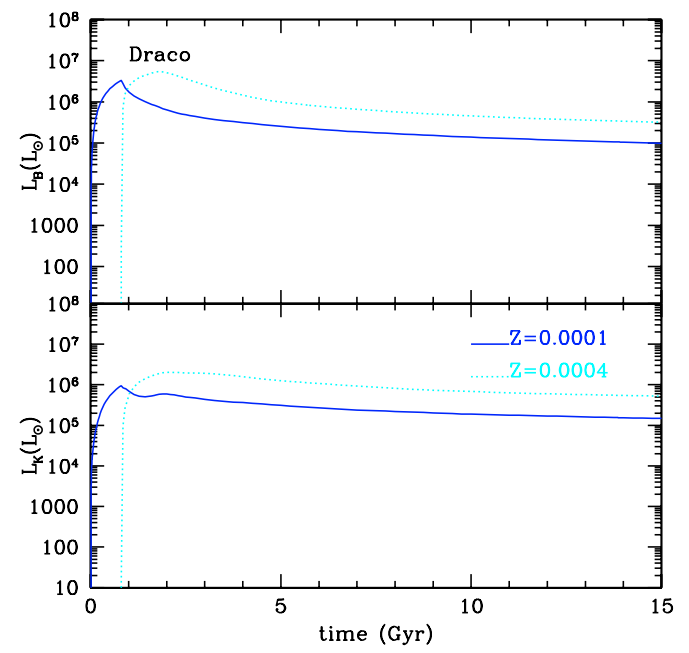

Fig. 6. Predicted $B$ - (upper panel) and $K$ - (lower panel) band luminosities for the different stellar populations of the Draco dSph formed at various metallicities as a function of time.

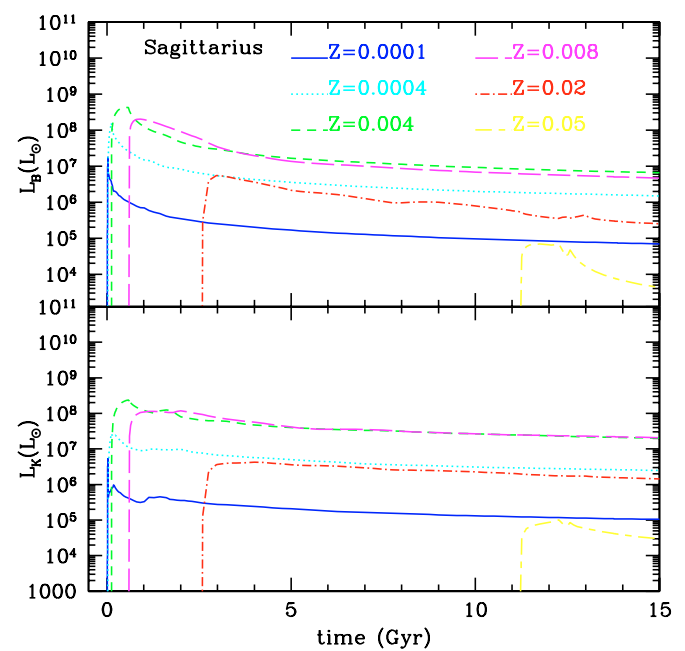

Fig. 7. Predicted $B$ - (upper panel) and $K$ - (lower panel) band luminosities for the different stellar populations of the Sagittarius dSph formed at various metallicities as a function of time.

by looking at the star formation histories (Fig. 1). The SFH of Draco peaks at $2 \mathrm{Gyr}$, corresponding to the age when the colour bump is largest in Fig. 9.

\subsection{The colour-magnitude relation: a comparison with giant ellipticals}

In Fig. 10, we plot the predicted present-day $U-V$ (lower panel) and $V-K$ (upper panel) colours as a function of the integrated absolute $V$ magnitude for the six dSphs studied in this paper (open squares). Our predictions are compared to the best fits (thick solid lines) of the colour-magnitude relations as observed by Bower et al. (1992), for the giant elliptical galaxies in the Coma and Virgo clusters. The dashed lines represent the $1 \sigma$ scatter to the data by Bower et al. (1992). For the early-type galaxies in the Virgo and Coma clusters, Bower et al. (1992) found a small scatter of $\sim 0.05$ in the colours as a function of the total $V$ magnitude. This small scatter implies a uniformity in the star formation histories of these systems, as well as a similar formation epoch (Renzini 2006). By comparing the data by Bower et al. (1992) to the colour-magnitude relations (CMRs) 


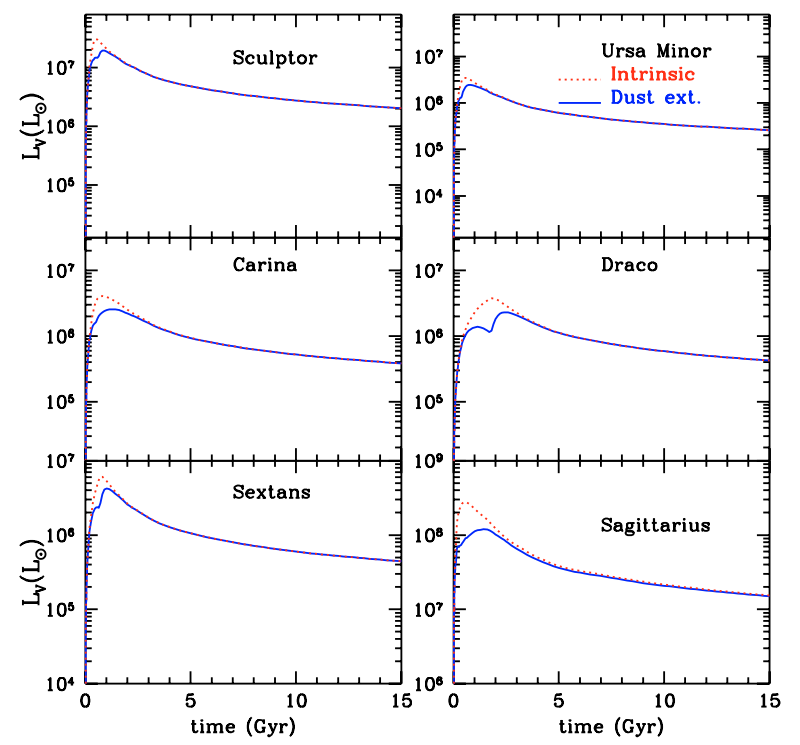

Fig. 8. Predicted intrinsic (dotted lines) and dust obscured (solid lines) total $V$ band luminosities for the six dSphs studied in this paper as functions of time.

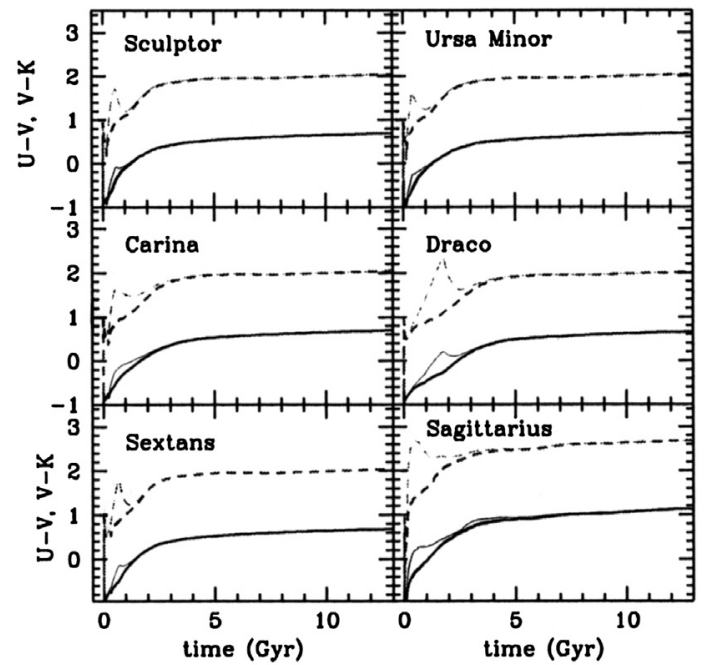

Fig. 9. Predicted $U-V$ (solid lines) and $V-K$ (dashed lines) colours for the six dSphs studied in this paper as functions of time. The thick lines represent the intrinsic predicted colours. The thin lines represent the colours calculated by taking into account dust extinction effects.

predicted for the dSphs, it is possible to see differences between the dSphs and giant ellipticals found in clusters, in particular concerning their star formation history. Furthermore, it is possible to test whether dwarf local spheroidals belong to the same class of objects, i.e. if the dSphs represent the low-mass tail of the mass function of a single population of galactic spheroids.

The CMR for cluster galaxies by Bower et al. (1992) has been determined for galaxies with maximum apparent $V$ magnitude 13 and $\sim 17$ in the case of the Virgo and Coma clusters, respectively. By adopting distance moduli of 31.1 and 35 for the Virgo (Freedman et al. 1994) and the Coma (Thomsen et al. 1997) clusters, respectively, the faintest absolute magnitudes which have been probed are $M_{V} \sim-18$, corresponding to galaxies considerably brighter than the local dSphs, presenting absolute magnitudes $M_{V} \leq-14$. For this reason, in Fig. 10, we are comparing the colours computed in this paper for the dSphs with the extrapolation of the Bower et al. (1992) relation.

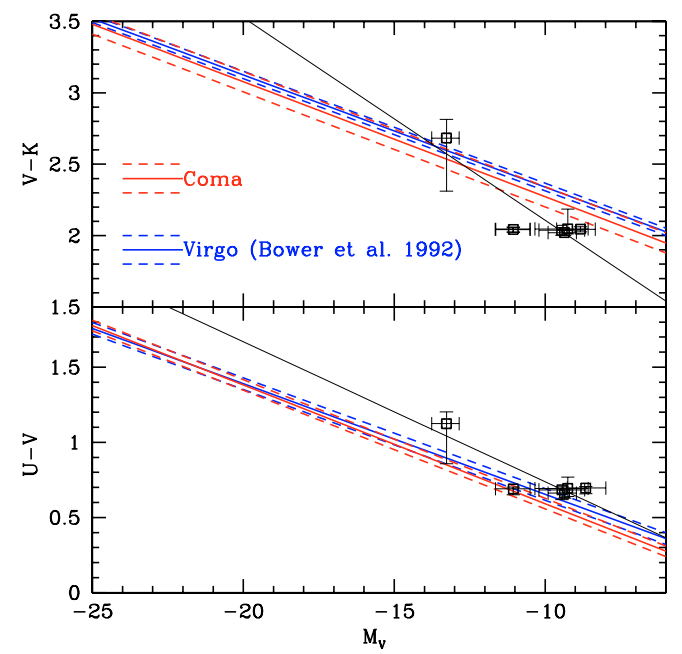

Fig. 10. Predicted present-day $U-V$ (lower panel) and $V-K$ (upper panel) colours for the six dSphs studied in this paper (open squares with error bars). The blue and red solid thick lines represent the fits of the Bower et al. (1992) data for early type galaxies in the Virgo and Coma clusters, respectively, with their $1 \sigma$ scatter (dashed thick lines). The thin lines represent the best fit to the predicted colours, computed by means of a straight line $y=a x+b$.

The most important aspect we are interested in is the slope of the CMR, since it is strictly linked to the galactic star formation history and age, in the sense that the tilt of the CMR tells us that fainter galaxies are bluer, hence have younger stellar populations. On the other hand, the scatter expresses how coeval the galaxies are in clusters and in the Local Group.

For each $\mathrm{dSph}$, the colour has been computed using the 3 models introduced in Sect. 2.1, i.e. by means of the fiducial model, the minimal model, and maximal model. This allows us to provide an error bar for the computed present-day colours of the six dSphs. By looking at the $U-V$ vs. $V$ plot (lower panel), we note that 5 out of $6 \mathrm{dSphs}$ show very similar colours. This is due to the fact that for all the six systems but Sagittarius, the bulk of the star formation was completed already several Gyr ago. Since 4 out 6 dSphs (Carina, Sextans, Ursa Minor and Draco) have very similar present-day luminosities and integrated absolute magnitudes (see Fig. 8), they tend to cluster in the same region of the $(U-V)$ vs. $V$ diagram. Sculptor is more luminous than these $4 \mathrm{dSphs}$, but presents similar present-day integrated colours. Sagittarius represents an exception, since it has a considerably higher luminosity and a redder colour.

In general, in the $(U-V)$ vs. $M_{V}$ plot, the dSphs are displaced to redder colours than cluster dwarfs of comparable luminosity. However, The predicted $(U-V)$ colours of the majority of the dSphs are compatible with the extrapolation to the faintest magnitudes of the colour-magnitude relation (CMR) for giant spheroids. We note that there is a partial overlap of the dSphs colours with the region including the scatter of the Virgo and Coma CMRs. In particular, the error bars of 5 (Carina, Sextans, Ursa Minor, Sculptor and Sagittarius) out of the 6 systems analyzed in this work fall within the dispersion found by Bower et al. (1992). The other system lies outside of the dashed lines and presents a slightly $(\sim 0.1 \mathrm{mag})$ redder $(U-V)$ than the cluster dwarf ellipticals of the same magnitude.

The $(V-K)$ colours of Carina, Sextans, Ursa Minor and Draco dSPhs are 0.2 mag bluer than the Coma spheroids of similar absolute magnitude, whereas Sculptor is 0.3 mag bluer. The 
$(V-K)$ colour of Sagittarius, plotted with its error bar, is compatible with the Virgo and Coma CMRs.

We plot also the best fits to the predicted CMR of the dSphs, represented by a straight line $y=a x+b$. From Fig. 10, it is clear that the slopes of he CMRs for dSphs and giant ellipticals are different. For the $(U-V)$ vs. $M_{V}$ relation for dSphs, the slope of the line which best fits our predicted values is $a_{\mathrm{dSph}}=-0.093$, against the values $a_{E, V}=-0.074 \pm 0.01$ and $a_{E, C}=-0.079 \pm$ 0.007 obtained by Bower et al. (1992) for the Virgo and Coma clusters, respectively.

From the study of the $(V-K)$ vs. $M_{V}$ plot, we find that $\mathrm{dSphs}$ have an even steeper CMR, with $a_{\mathrm{dSph}}=-0.14$, against $a_{E, V}-0.078 \pm 0.007$ and $a_{E, C}-0.08 \pm 0.013$. The differences between the CMRs of giant ellipticals and dSphs, in particular concerning the $(V-K)$ vs. $V$ plot, are very likely due to a more protracted SFHs of the dSphs with respect to the ones of the cluster ellipticals. In fact, the CMDs of dSphs indicate that in general, they have experienced long star formation histories, lasting several Gyr (see Fig. 1, Aparicio et al. 2001; Rizzi et al. 2003), at variance with their giant counterparts, which formed the bulk of their stars in 0.2-0.5 Gyr. In the case of the giant ellipticals, the scatter of the CMR as derived by Bower et al. (1992) implies a dispersion in the age of the spheroid stellar populations of $\Delta_{\text {form }} \sim 2$ Gyr. Although presenting similar present-day colours, the dSphs present a larger dispersion in the age of their stellar populations: for instance, the SFH of the Ursa Minor dSph is completed after $3 \mathrm{Gyr}$, whereas Carina undergoes star formation episodes until 10 Gyr of evolution (see Fig. 1). This implies that the formation of dSphs occurred with a lower degree of synchronicity than the formation of cluster ellipticals. Although presenting a larger dispersion in the ages, in the colour-magnitude plots it is not unlikely that the local dSphs studied here form a sequence similar to the one observed for their giant counterparts in clusters. The apparent differences between the colours of local dSphs and cluster dwarfs may be due to environmental effects: the clusters are in general denser environments, where interactions with the intra-cluster medium or with other galaxies are more frequent than in the Local Group and may have some effects also on the photometric evolution of their members. By means of cosmological simulations, Bullock et al. (2001) showed that dark matter halos in denser environments form earlier than more isolated halos. For this reason, cluster dwarfs may form earlier or may evolve faster, showing at the present day redder colours than local dSphs.

Since the sample considered here is limited to six systems, from the current analysis it is not possible to draw firm conclusions on this issue, i.e. whether local dSphs may represent the low-mass tail of giant ellipticals. In the future, it will be important to extend our analysis to a larger number of dSphs, in order to improve the statistics, as well as to extend observational surveys in clusters to fainter absolute magnitudes. In the next Section, we will discuss implications of our analysis for the formation of spheroids.

\subsection{Implications for the formation of the giant galaxies}

An important question concerns the possibility that dSphs could represent the building blocks of the local giant ellipticals. Chemical evolution studies indicate that the dSphs are unlikely to represent the building blocks of giant spiral galaxies. This is suggested by the abundance patterns observed in most of the dSph stars, which are clearly distinct from the ones observed in the stars of the Milky Way halo and disc (Venn et al. 2004). This fact represents a challenge to all galaxy formation models based on the popular $\Lambda$ cold dark matter $(\Lambda C D M)$ cosmological scenario. According to these models, in a $\Lambda$ CDM-dominated universe, small dark matter halos are the first to collapse, then interact and merge to form larger halos. In this framework, massive spheroids are formed from several merging episodes among smaller objects (Somerville et al. 2001; Menci et al. 2002). This scenario for the most massive galaxies is at variance with the old monolithic collapse scenario, where ellipticals and bulges formed at high redshift (e.g. $z>2-3$ ) as the result of a violent burst of star formation following a "monolithic collapse" of a gas cloud (Larson 1974; Sandage 1986; Arimoto \& Yoshii 1987; Matteucci 1994).

A picture where small and large spheroids form quasimonolithically, although with different star formation timescales, accounts naturally for the fact that local dwarf spheroidals may represent the low mass counterparts of giant ellipticals. A possible link between dSphs and giant ellipticals was suggested by Tamura et al. (2001), who showed that both the mass-metallicity and mass-luminosity relations of some $\mathrm{d}$ Sph galaxies may be understood as a low-mass extension of giant elliptical galaxies.

The link between dSphs and giant ellipticals is supported by the scenario proposed by Zaritsky et al. (2006), who found that small and giant spheroids belong to the same family and that a "fundamental manifold" for spheroids of all masses exist, i.e. that the local spheroids of all masses follow the same fundamental plane, with very few exceptions. As argued also by Zaritsky et al. (2006), it is probably difficult to account for the tightness of the CMR and of the spheroid manifold in a hierarchical framework, where the dSphs are the few survivors or intense merging activity. In the hierarchical paradigm, the dwarf spheroidal galaxies should be the first galaxies to form their stars. The giant galaxies should assemble later by progressive merging of smaller systems. As a consequence, in general the dwarf galaxies should show stellar populations redder than their giant counterparts. A scenario in which dSphs were the building blocks of giant ellipticals would give rise to a CMR for dwarf and giant spheroids characterised by a shallower slope and a larger dispersion than what the observations indicate, as shown by Kauffmann \& Charlot (1998) by means of semi-analythical models and by Saro et al. (2006) by means of numerical simulations. These papers showed that, in a scenario where the galaxies grow hierarchically, the present-day tilt and tightness of the CMR represents one of the most challenging features to explain.

Other open questions in cosmological studies of dSphs concern the "missing satellite" problem, i.e. the discrepancy between the observed number of dwarf galaxies clustered around giant spirals and the numbers predicted by hierarchical models (Moore et al. 1999) and their anisotropical spatial distribution (Kroupa et al. 2005), difficult to account for by current $\Lambda C D M$ models. Together with the arguments studied in this section, these points outline our incomplete understanding of the process of galaxy formation.

In Table 3, we present a summary of some of the observed and predicted photometric properties of the dSph galaxies studied in this work. In Col. 1, we list all the six dwarf spheroidal galaxies. In Cols. 2 and 3, we present the observed and predicted present-day integrated $(B-V)$ colours, respectively. In Cols. 4 and 5, we present the observed and predicted present-day HI to blue luminosity ratios $\left(M_{\mathrm{HI}} / L_{\mathrm{B}}\right)$, respectively.

For the present-day $(B-V)$ values of Sculptor, Carina, Sextans, Ursa Minor and Sagittarius we use the observational data published by Mateo (1998). Some of these data are presented without error bars. For Draco dSph, we also use the 
Table 3. Photometric properties of Local Group Dwarf galaxies.

\begin{tabular}{lcccc}
\hline \hline Galaxy & \multicolumn{2}{c}{$(B-V)$} & \multicolumn{2}{c}{$M_{\mathrm{HI}} / L_{\mathrm{B}}$} \\
& Obs & Pred & Obs & Pred \\
\hline Sculptor & $0.7^{1}$ & $0.67 \pm 0.02$ & $0.01^{1}$ & $0.0006-0.0018$ \\
Carina & $0.7^{1}$ & $0.67 \pm 0.05$ & $<0.002^{1}$ & $0.008-0.022$ \\
Sextans & $0.7^{1}$ & $0.66 \pm 0.04$ & $<0.001^{1}$ & $0.001-0.005$ \\
Ursa Minor & $1.3 \pm 0.3^{1}$ & $0.67 \pm 0.02$ & $<0.002^{1}$ & $0.007-0.017$ \\
Draco & $0.95 \pm 0.2^{1} ; 0.5 \pm 0.7^{2}$ & $0.65 \pm 0.02$ & $<0.007^{1,2}$ & $0.008-0.036$ \\
Sagittarius & $0.6^{1}$ & $0.8 \pm 0.01$ & $<0.001^{1}$ & $0.0012-0.0016$ \\
\hline
\end{tabular}

Note: ${ }^{1}$ Mateo (1998); ${ }^{2}$ Aparicio et al. (2001).

observational $B, V$ magnitudes and $M_{\mathrm{HI}} / L_{\mathrm{B}}$ as published by Aparicio et al. (2001). By considering the error of $0.2-0.3$ given by Mateo (1998) for the $(B-V)$ colours of Ursa Minor and Draco as representative for all the other systems, we note that the $B$ and $V$ magnitudes of all the galaxies considered here are reproduced with good accuracy, with one exception. According to the data by Mateo (1998), the Ursa Minor dSph is considerably redder than what we predict, with a discrepancy between our predictions and the observed $(B-V)$ of $0.6 \mathrm{mag}$. Interestingly, the observed $(B-V)$ is considerably redder even than giant ellipticals in local clusters. In fact, in the Fornax cluster, Karick et al. (2003) observe for elliptical galaxies $(B-V)$ ranging from $0.6 \mathrm{mag}$ up to $1.1 \mathrm{mag}$. This tells us that the star formation history of the Ursa Minor was probably more complex than the one modelled in this paper. Moreover, a recent large-area photometric survey of the Ursa Minor dSph showed a peculiar structure of this galaxy, with an elongated morphology possibly due to tidal interaction with the Milky Way (Bellazzini et al. 2002). In the light of these results, Bellazzini et al. (2002) recomputed the integrated $V$ magnitude of Ursa Minor finding $M_{V}=-10.3 \pm$ 0.4 , against the value $M_{V}=-8.9$ computed by Mateo (1998) without taking account any distortion effect. Unfortunately, no up-to-date estimations are available for the corrected integrated $B$ magnitude. We await further data able to confirm or to rule out the peculiar $(B-V)$ colour of the Ursa Minor dSph.

As shown in Table 3, in most of the cases, a scenario like ours, where the bulk of the dSphs stars formed prior to any interactions among them and with their largest conterparts, is able to account for the observed integrated colours of the local dSphs. Throughout this paper, we have assumed that the interactions between the dSphs and the external environment and with the giant galaxies of the Local Group had negligible effects on their stellar populations, in the sense that they must have occurred after the bulk of the stars were formed. However, it is important to note that effects such as tidal interactions with the Milky Way may play some role in the evolution of dSphs. The most striking example is represented by the Sagittarius $\mathrm{dSph}$, which is characterised by a tidal tail extending from its centre out to a distance of 20-40 kpc (Mateo et al. 1996; Majewski et al. 2003). In this case, such an interaction may have triggered a star formation episode a few Gyr ago, as indicated by the $\mathrm{M}$ giants observed in its stream. Another case is represented by Carina. For this galaxy, photometric studies of regions beyond the classical tidal radius show a stellar distribution extending as far as several degrees from its centre (Kuhn et al. 1996; Muñoz et al. 2006) All of these results indicate that the main effects of these interactions affected the last few Gyr of evolution and were mainly of a dynamical nature (see Klimentowski et al. 2006), without affecting significantly the past star formation history of the dSphs.

On the other hand, for most of the dSphs we overestimate the observed $\left(M_{\mathrm{HI}} / L_{\mathrm{B}}\right)$, with the exception of the Sculptor dSph.
This is again due to the fact that our models do not include interactions with the external environment, in this case dynamical gas removal mechanisms, such as ram pressure stripping or tidal interactions. These dynamical processes, beside galactic winds, are likely to play a non negligible role in sweeping the gas from the dSph galaxies (Grebel et al. 2003). To investigate the effects of ram pressure stripping, hydrodynamical simulation studies should be required (see Marcolini et al. 2006).

In the case of the Sculptor dSph, we severely underestimate the observed $\left(M_{\mathrm{HI}} / L_{\mathrm{B}}\right)$ reported by Mateo (1998). Observations of this galaxy indicate that it presents a particularly high content of HI. A possible explanation is that the Sculptor dSph is rapidly accreting gas from the external clouds, as its proximity to high velocity clouds is most likely suggesting (Carignan et al. 1998, 1999).

\subsection{What do the colours tell us of the evolutionary history of dSph galaxies?}

An interesting question concerning dSphs is how their SFHs have proceeded in the past. To investigate this aspect, of particular interest is the study of the relationship between dSphs and dwarf irregulars which could represent the same objects seen in different evolutionary phases. In general, dSphs have physical properties rather different than dIrrs (Grebel et al. 2003, and references therein): (1) the gas content, with dSphs being gas-poor, whereas dIrrs contain large reservoirs of gas; (2) rotation: dSphs have little angular momentum, whereas dIrrs are fast rotating systems; (3) metallicity: dSphs present metallicities in general higher than dIrrs at the same optical luminosity. All these arguments stand against a continuous evolution from dIrrs to dSphs and seem to indicate that they represent systems with different origin and past SFHs.

LM03 analyzed a possible connection between the $\mathrm{dSph}$ galaxies and Blue Compact Dwarf Galaxies (BCGs). The SFHs inferred from the CMDs of BCGs are characterised by short starbursts separated by periods of inactivity (Schulte-Ladbeck et al. 2001), similar to the ones derived from the CMDs of dIrr galaxies (Kunth \& Östlin 2000; Annibali et al. 2003). LM03 claimed that the chemical evolution of BCGs and $\mathrm{dSphs}$ indicates marked differences in their SFHs. While the observed distributions of several abundance ratios in BCGs are reproduced by models with SF characterised by short and repeated bursts separated by long quiescent periods, the abundance ratios of local dSph galaxies require a SF which proceeds in one long episode of low activity. The SF efficiencies of the two models are basically different, as well as the efficiencies of the galactic winds, which are much higher $\left(w_{i}=4-13\right)$ for the dSphs than in the case of the BCGs $\left(w_{i}=0.25-1\right)$. They also concluded that a bursting model, suited to describe the SFHs of both dIrrs and BCGs, is not able to reproduce the dSphs data and vice-versa. 


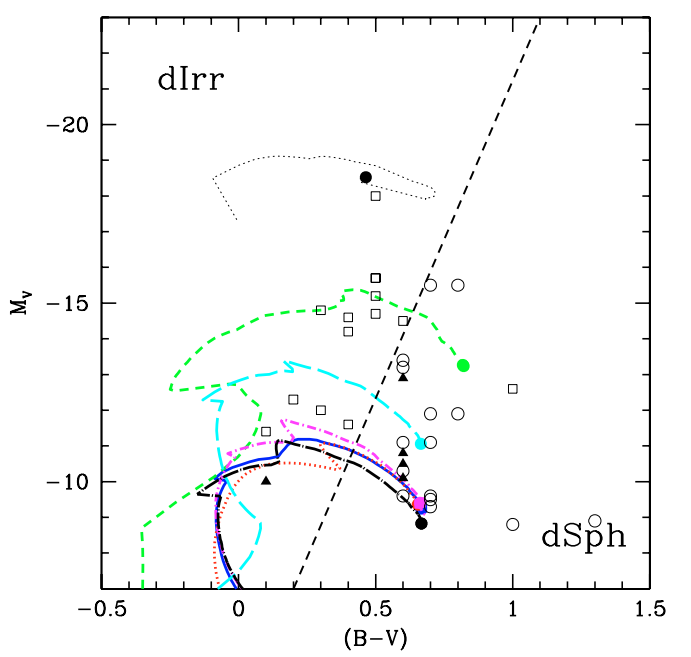

Fig. 11. Observed and predicted $V$ magnitude vs. $(B-V)$ colours for various dwarf galaxies. The thick solid lines represent the evolution of the $V$ magnitudes as a function of the $(B-V)$ colour for the six dSphs studied in this work (solid line: Carina; dotted line: Draco; short-dashed line: Sagittarius; long-dashed line: Sculptor; dot-short-dashed: Sextans; dot-long-dashed: Ursa Minor). The solid dots at the end of each line represent the present-day values. The dotted thin line represents the predictions obtained by means of a model for the Large Magellanic cloud. The open circles, open squares and solid triangles are the observed values for dSph galaxies, dIrrs and transition objects, respectively (data from Mateo 1998). The dashed line is the same as the one drawn in Fig. 5 of Mateo (1998) and separates the systems classified as dIrrs (left region of the plot) and dSphs (right region).

However, some other evidence is in favour of a possible transition from dIrrs to dSphs, in particular, (a) the similarity of the exponential radial brightness profiles and, (b) the existence of the "transition type" dwarf galaxies, i.e. systems presenting properties common to both kinds of objects, such as old stellar populations (typical of dSphs) and high gas content (typical of dIrrs). Some important indications of the past evolution of dwarf galaxies come from the study of their integrated absolute $V$ magnitude as a function of their $(B-V)$ colour (Mateo 1998). In Fig. 11, we plot the predicted evolution of the integrated $V$ magnitude as a function of the $(B-V)$ colour for the six dwarf spheroidal galaxies studied in this work (solid thick lines). The dotted thin line indicates the evolution of the $M_{V}$ vs. $(B-V)$ for a model representing the Large Magellanic Cloud (LMC). This model is the same as the one used by Calura et al. (2003) to study the chemical evolution of the Damped Lyman Alpha absorbers. For further details on this model, we refer the reader to that paper. This model is used to represent the evolution of the $V$ magnitude as a function of the $(B-V)$ colour for a typical dIrr galaxy. The solid circles at the end of each line represent the present-day values.

Along with the predicted values, in Fig. 11 we plot also the observed values for three different kinds of objects belonging to the Local Group (Mateo 1998): the dSph galaxies (open circles), the dIrrs (open squares) and the transition objects (solid triangles). The dashed line is the same as the one drawn in Fig. 5 of Mateo (1998) and it is helpful to show how, in this plot, the dIrrs tend to be segregated from the dSphs. The transition objects are in general located close to the dashed line. It is interesting to see how, in general, at the same $V$ magnitude, dIrrs tend to be bluer than dSph galaxies. Our predictions indicate that, in their past history, when they were bluer and more gas-rich than today, the $\mathrm{dSph}$ galaxies used to lay on the left of the dashed line, in the

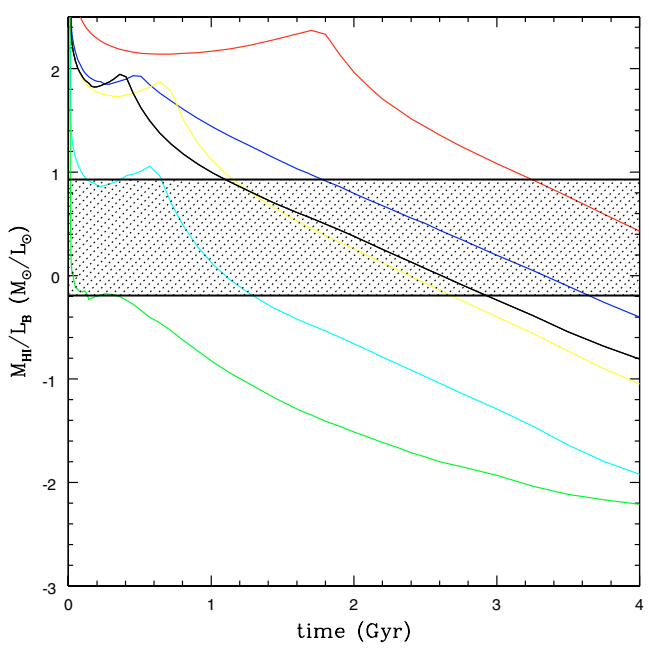

Fig. 12. Evolution of $M_{\mathrm{HI}} / L_{\mathrm{B}}$ four the six dSphs (solid lines) during their initial $4 \mathrm{Gyr}$ of evolution, corresponding approximately to the time that each dSph galaxy spends in the left part of the plot in Fig. 11. The shaded area encloses all the possible values for $M_{\mathrm{HI}} / L_{\mathrm{B}}$ observed in local dIrr (Mateo 1998).

region where the present day dIrrs lie. This is visible in the left part of the plot, where the solid thick lines overlap with the open squares. According to our results, during their past history, the dSphs may have shown photometric properties similar to those of the present day dIrrs.

Our results show that dSphs and dIrrs may share a common progenitor phase, as pointed out also by Binggeli (1994) and Skillmann \& Bender (1995). During this phase, both dSphs and dIrrs may have occupied the left region of $V$ vs. $(B-V)$ plot and then they may have evolved through different branches. Since then, dIrrs have experienced little evolution and they have not reached the right side of the $M_{V}$ vs. $(B-V)$ plot. This seems to be suggested also by the predicted evolution of $V$ vs. $(B-V)$ for the $\mathrm{LMC}^{1}$ galaxy in Fig. 10.

On the other hand, dSphs have experienced a stronger evolution and they have moved to the right part of the $V$ vs. $(B-V)$ plot. It is nevertheless possible that a sub-class of dSphs may derive from dIrrs. The transition galaxies may represent this sub-class of objects, which are presumably younger than the dSphs.

Finally, it is interesting to see whether in the past, the gas content of dSphs was compatible with what local observations of dIrrs indicate. In Fig. 12, we plot the time evolution of $M_{\mathrm{HI}} / L_{\mathrm{B}}$ for the six dSphs during their initial 4 Gyr of evolution, corresponding approximately to the time that each $\mathrm{dSph}$ galaxy spends in the left part of the plot in Fig. 11, which can be regarded as the "dIrr" phase. The shaded area encloses all the possible values for $M_{\mathrm{HI}} / L_{\mathrm{B}}$ observed in local dIrr as published by Mateo (1998). We see that, for all dSphs with the exception of one case (Sagittarius), the phase when $M_{\mathrm{HI}} / L_{\mathrm{B}}>0.64 M_{\odot} / L_{\odot}$ (corresponding to the lowest $M_{\mathrm{HI}} / L_{\mathrm{B}}$ observed in dIrrs, Mateo 1998) lasts more than 1 Gyr. In Fig. 12, the region bracketed by the shaded area is only indicative of the past $M_{\mathrm{HI}} / L_{\mathrm{B}}$ values for dIrrs, since in the past dIrrs are likely to have shown $M_{\mathrm{HI}} / L_{\mathrm{B}}$ values larger than the current ones. This confirms that during their

1 Notwithstanding the large mass of the LMC, which renders it probably too massive to be regarded as an average dIrr galaxy, its evolution in the $M_{V}$ vs. $(B-V)$ plot shows that it never crossed the dashed line. For this reason, from the photometric point of view, in this plot it can be regarded as an example of a typical dIrr. 
first few Gyr of evolution, the majority of dSphs would have looked like the dIrr as we see them today.

\section{Summary and conclusions}

By means of a chemical evolution model combined with a spectro-photometric code we were able to predict the evolution of several photometric properties of six Local Group Dwarf Spheroidal galaxies (Carina, Draco, Sagittarius, Sculptor, Sextans and Ursa Minor). The chemical evolution models adopt up-to-date nucleosynthesis from intermediate mass stars, massive stars and SNe type II and Ia and take into account the contribution of SNe to the energetics of the ISM. For the six $\mathrm{dSphs}$, the star formation histories are taken from the observed colour-magnitude diagrams (CMDs, Dolphin et al. 2005). The proposed scenario for the evolution of these galaxies is characterised by low star formation rates and high galactic wind efficiencies. Such a scenario allows us to predict colours and magnitudes in agreement with observations.

The main conclusions can be summarized as follows:

- The total luminosities of 5 (Sculptor, Carina, Sextans, Ursa Minor and Draco) out of $6 \mathrm{dSphs}$ galaxies analysed here are dominated by the SSPs formed at the lowest metallicities, i.e. $Z \leq 0.0004$. This is a consequence of the low SF efficiency and of the intense wind which removes a large fraction of the gas of the galaxy, almost halting the SF and preventing metal-rich stars to be formed. For each galaxy, the stellar populations dominating the metallicity distribution (LM04) dominate also the total light.

- In the case of Sagittarius, the $B$ and $K$ luminosities are dominated by stellar populations with higher metallicities, i.e. $Z \geq 0.004$, due to the much higher SF efficiency adopted for this galaxy ( $>10$ times higher than the ones of the other dSphs).

- Only during the SF phase, when all galaxies exhibit a high gas content, the effects of dust extinction in the total luminosities in the $U, V, B$ bands are noticeable. After the onset of the galactic winds and the consequent removal of the interstellar gas, the effects of extinction become negligible.

- We compared the predicted current integrated $(U-V)$ and $(V-K)$ colours as a function of the absolute $V$ magnitude for the $\mathrm{dSphs}$ to the colour-magnitude relation for giant spheroids in clusters. The main differences between dSphs and the giant ellipticals concern their star formation histories. In particular, the formation of dSphs occurred with a lower degree of synchronicity than the formation of giant ellipticals. The differences may also be due to environmental effects, since clusters are environments denser than the Local Group. In the colour-magnitude plots, the six local $\mathrm{dSphs}$ studied here seem to form a sequence similar to the one observed for their counterparts in clusters, although with a slightly steeper slope and a larger dispersion in their ages. Although we can not draw any firm conclusion on this aspect, it is not unlikely that the dSphs can be regarded as the lowest-mass tails of their larger counterparts and not the building blocks from which they were assembled. This issue represents a challenge to all galaxy formation models based on the popular $\Lambda$ cold dark matter cosmological scenario.

- The study of the evolution of the integrated $V$ magnitude as a function of the $(B-V)$ colour suggests that, during their past history, the dSphs may have shown photometric properties similar to the ones of the present-day dIrrs. It is possible that $\mathrm{dSphs}$ and dIrrs shared a common progenitor phase, and then evolved through different paths. After the common phase, dIrrs have experienced little evolution, mantaining most of their gas and blue colours. dSphs have instead experienced a stronger evolution, losing all of their gas and showing present-time colours redder than dIrrs. Transition types might represent a sub-class of dIrrs, which may evolve into dSphs.

Acknowledgements. We wish to thank Simone Recchi for a critical reading of the manuscript and for several useful comments and suggestions. We are grateful to an anonymous referee for several useful comments that improved the quality of our work. G.A.L. thanks the hospitality of the Dipartimento di Astronomia-Universitá di Trieste which provided all the support during his stay. G.A.L. acknowledges financial support from the Brazilian agency FAPESP (proj. 06/57824-1). F.M. and F.C. acknowledge financial support from INAF (Italian National Institute of Astrophysics) project 2005_1.06.08.16.

\section{References}

Annibali, F., Greggio, L., Tosi, M., Aloisi, A., \& Leitherer, C. 2003, AJ, 126, 2752

Aparicio, A., Carrera, R., \& Martinez-Delgado, D. 2001, AJ, 122, 2524

Arimoto, N., \& Yoshii, Y. 1987, A\&A, 173, 23

Bellazzini, M., Ferraro, F. R., Origlia, L., et al. 2002, AJ, 124

Binggeli, B. 1994, in Panchromatic View of Galaxies, ed. G. Hensler, Ch. Theis, \& J. Gallagher, 173

Bonifacio, P., Hill, V., Molaro, P., et al. 2000, A\&A, 359, 663

Bonifacio, P., Sbordone, L., Marconi, G., Pasquini, L., \& Hill, V. 2004, A\&A, 414,503

Bower, R. G., Lucey, J. R., \& Ellis, R. S. 1992, MNRAS, 254, 613

Bradamante, F., Matteucci, F., \& D'Ercole, A. 1998, A\&A, 337, 338

Bruzual, A. G., \& Charlot, S. 2003, MNRAS, 344, 1000

Bullock, J. S., Kolatt, T. S., Sigad, Y., et al. 2001, MNRAS, 321, 559

Calura, F., \& Matteucci, F. 2004, MNRAS, 350, 351

Calura, F., \& Matteucci, F. 2006, ApJ, 652, 889

Calura, F., Matteucci, F., \& Vladilo, G. 2003, MNRAS, 340, 59

Calura, F., Matteucci, F., \& Menci, N. 2004, MNRAS, 353, 500

Calzetti, D. 1997, in The Ultraviolet Universe at Low and High Redshift: Probing the Progress of Galaxy Evolution, ed. W. H. Waller et al., AIP Conf. Proc., 408,403

Calzetti, D. 2001, PASP, 113, 1449

Calzetti, D., Kinney, A. L., \& Storchi-Bergmann, T. 1994, ApJ, 429, 582

Carignan, P. 1999, PASA, 16, 18

Carignan, C., Beaulieu, S., Côté, S., et al. 1998, AJ, 116, 1690

Carrera, R., Aparicio, A., Martinez-Delgado, D., \& Alonso-Garcia, J. 2002, AJ, 123,3199

Cescutti, G., François, P., Matteucci, F., Cayrel, R., \& Spite, M. 2006, A\&A, 448,557

Côté, S., Freeman, K. C., Carignan, C., \& Quinn, P. J. 1997, AJ, 114, 1313

Davies, J. I., \& Phillips, S. 1988, MNRAS, 233, 553

Dekel, A., \& Silk, J. 1986, ApJ, 303, 39

Dolphin, A. E. 2002, MNRAS, 332, 91

Dolphin, A. E., Weisz, D. R., Skillman, E. D., \& Holtzman, J. A. 2005, Resolved Stellar Populations, ASP Conf. Ser., ed. D. Valls-Gabuad, \& M. Chavez [arXiv: astro-ph/0506430]

Fenner, Y., Gibson, B. K., Gallino, R., \& Lugaro, M. 2006, ApJ, 646, 184

Ferguson, H. C., \& Sandage, A. 1991, AJ, 101, 765

François, P., Matteucci, F., Cayrel, R., et al. 2004, A\&A, 421, 613

Freedman, W. L., Madore, B. F., Mould, J. R., et al. 1994, Nature, 371, 757

Ikuta, C., \& Arimoto, N. 2002, A\&A, 391, 55

Gallagher, J. S., Madsen, G. J., Reynolds, R. J., Grebel, E. K., \& Smecker-Hane, T. A. 2003, ApJ, 588, 326

Geisler, D., Smith, V. V., Wallerstein, G., Gonzalez, G., \& Charbonnel, C. 2005, AJ, 129, 1428

Geisler, D., Wallerstein, G., Smith, V. V., \& Casetti-Dinescu, D. I. 2007, PASP, 119, 939

Grebel, E. K. 1998, in Highlights of Astronomy, ed. J. Andersen (Kluwer, Dordrecht), 11, 125

Grebel, E. K. 2001, in Dwarf Galaxies and Their Environment, ed. K. S. De Boer, R. Dettmar, \& U. Klein (Aachen: Shaker Verlag), 45

Grebel, E. K., Gallagher, J. S., III, \& Harbeck, D. 2003, AJ, 125, 1926

Greggio, L., \& Renzini, A. 1983, A\&A, 118, 217 
Graham, A., \& Guzm‘an, R. 2003, ApJ, 2936

Guzm‘an, R., Jangren, A., Koo, D. C., Bershady, M. A., \& Simard, L. 1998, ApJ, L13

Hernandez, X., Gilmore, G., \& Valls-Gabaud, D. 2000, MNRAS, 317, 831

Hurley-Keller, D., Mateo, M., \& Nemec, J. 1998, AJ, 115, 1840

Irwin, M., \& Hatzidimitriou, D. 1995, MNRAS, 277, 1354

Karick, A. M., Drinkwater, M. J., \& Gregg, M. D. 2003, MNRAS, 344, 188

Kauffmann, G., \& Charlot, S. 1998, MNRAS, 294, 705

Kawata, D., Arimoto, N., Cen, R., \& Gibson, B. K. 2006, ApJ, 641, 785

Klimentowski, J., Lokas, E. L., Kazantzidis, S., et al. 2006, MNRAS, submitted [arXiv:astro-ph/0611296]

Koch, A., Grebel, E. K., Wyse, R. F. G., et al. 2005, AJ, 131, 895

Koch, A., Grebel, E. K., Wyse, R. F. G., et al. 2006, AJ, 131, 895

Kroupa, P., Theis, C., \& Boily, C. M. 2005, A\&A, 431, 517

Kuhn, J. R., Smith, H. A., \& Hawley, S. L. 1996, ApJ, 469, L93

Kunth, D., \& Östlin, G. 2000, A\&AR, 10, 1

Lanfranchi, G., \& Matteucci, F. 2003, MNRAS, 345, 71, LM03

Lanfranchi, G., \& Matteucci, F. 2004, MNRAS, 351, 1338, LM04

Lanfranchi, G., \& Matteucci, F. 2007, A\&A, 468, 927, LM07

Lanfranchi, G., Matteucci, F., \& Cescutti, G. 2006a, MNRAS, 365, 477

Lanfranchi, G., Matteucci, F., \& Cescutti, G. 2006b, A\&A, 453, 67

Larson, R. B. 1974, MNRAS, 166, 585

Lin, D. N. C., \& Faber, S. M. 1983, ApJ, 266, L21

Majewski, S. R., Skrutskie, M. F., Weinberg, M. D., \& Ostheimer, J. C. 2003, ApJ, 599, 1082

Marcolini, A., D'Ercole, A., Brighenti, F., \& Recchi, S. 2006, MNRAS, in press

Mateo, M. L. 1998, ARA\&A, 36, 435

Mateo, M., Mirabal, N., Udalski, A., et al. 1996, ApJ, 458, L13

Matteucci, F. 1992, ApJ, 397, 32

Matteucci, F. 1994, A\&A, 288, 57

Matteucci, F. 1996, FCPh, 17, 283

Matteucci, F., \& Greggio, L. 1986, A\&A, 154, 279

Matteucci, F., \& Tornambé, A. 1987, A\&A, 185, 5

Mayer, L., Mastropietro, C., Wadsley, J., Stadel, J., \& Moore, B. 2006, MNRAS, 369,1021

Menci, N., Cavaliere, A., Fontana, A., Giallongo, E., \& Poli, F. 2002, ApJ, 575, 18

Moore, B., Ghigna, S., Governato, F., et al. 1999, ApJ, 524, L19
Monaco, L., Bellazzini, M., Bonifacio, P., et al. 2005, A\&A, 441, 141 Mori, M., \& Burkert, A. 2000, ApJ, 538, 559

Muñoz, R. R., Majewski, S. R., Zaggia, S., et al. 2006, ApJ, 649, 201

Nomoto, K., Hashimoto, M., Tsujimoto, T., et al. 1997, Nucl. Phys. A, 616, 79c Papaderos, P., Loose, H.-H., Fricke, K. J., \& Thuan, T. X. 1996, A\&A, 314, 59 Phillipps, S., Driver, S. P., Couch, W. J., \& Smith, R. M. 1998, ApJ, 498, L119 Recchi, S., Matteucci, F., D’Ercole, A., \& Tosi, M. 2002, A\&A, 384, 799 Renzini, A. 2006, ARA\&A, 44, 141

Ricotti, M., \& Gnedin, N. Y. 2005, ApJ, 629, 259

Rizzi, L., Held, E. V., Bertelli, G., \& Saviane, I. 2003, ApJ, 589, L85

Sadakane, K., Arimoto, N., Ikuta, C., et al. 2004, PASJ, 56, 1041

Salpeter, E. E. 1955, ApJ, 121, 161

Sandage, A. 1986, A\&A, 161, 89

Saro, A., Borgani, S., Tornatore, L., et al. 2006, MNRAS, 373, 397

Shapley, H. 1938, Harvard College Obs. Bulletin, 908, 1

Smecker-Hane, T. A., Stetson, P. B., Hesser, J. E., \& Vandenberg, D. A. 1996, in From Stars to Galaxies: The Impact of Stellar Physics on Galaxy Evolution, ed. C. Leitherer, U. Fritze-von-Alvensleben, \& J. Huchra, ASP Conf. Ser., 98, 328

Schmidt, M. 1963, ApJ, 137, 758

Schulte-Ladbeck, R. E., Hopp, U., Greggio, L., Crone, M. M., \& Drozdovsky, I. O. 2001, Ap\&SS, 277, 309

Shetrone, M., Côté, P., \& Sargent, W. L. W. 2001, ApJ, 548, 592

Shetrone, M., Venn, K. A., Tolstoy, E., \& Primas, F. 2003, AJ, 125, 684

Skillman, E. D., \& Bender, R. 1995, RMxAC, 3, 25

Somerville, R. S., Primack, J. R., \& Faber, S. M. 2001, MNRAS, 320, 504

Tamura, N., Hirashita, H., \& Takeuchi, T. T. 2001, ApJ, 552, L113

Thielemann, F. K., Nomoto, K., \& Hashimoto, M. 1996, ApJ, 460, 408

Thomsen, B., Baum, W. A., Hammergren, M., \& Worthey, G. 1997, ApJ, 483, L37

Tinsley, B. M. 1980, FCPh, 5, 287

Tolstoy, E., Venn, K. A., Shetrone, M., et al. 2003, AJ, 125, 707 Tolstoy, E., Irwin, M. J., Helmi, A., et al. 2004, ApJ, 617, L119

van den Bergh, S. 1994, ApJ, 428, 617

Van den Hoeck, L. B., \& Groenwegen, M. A. T. 1997, A\&AS, 123, 305

Venn, K. A., Irwin, M., Shetrone, M. D., et al. 2004, AJ, 128, 1177

Woosley, S. E., \& Weaver, T. A. 1995, ApJS, 101, 181

Zaritsky, D., Gonzalez, A. H., \& Zabludoff, A. I. 2006, ApJ, 642, L37 